\title{
Supporting people with diabetes during the COVID-19 pandemic without face-to-face appointments
}

\author{
DINESH NAGI, ${ }^{1}$ PRATIK CHOUDHARY, ${ }^{2}$ EMMA G WILMOT, ${ }^{3}$ PETER WINOCOUR ${ }^{4}$
}

\begin{abstract}
"We've been in full \#COVID19 mode in my hospital in Alcorcón (Madrid), Spain for the past 2 weeks. This crisis has forced us to adapt like we never thought possible. \#FOAMcovid."
\end{abstract}

Miguel Galan de Juana

Key words: COVID-19, type 1 Diabetes, type 2 Diabetes, virtual consultation, E-consultation

We write this article deep during the coronavirus pandemic gripping the UK. While we are not anywhere near through with this, it would appear that perhaps it is the end of the beginning. The first phase of an attack by the unseen enemy called COVID-19 has killed over 350,000 people all over the world. None of us imagined that we would be in the dire straits we find ourselves when we first heard about a deadly coronavirus case in China on 31 December 2019. Several weeks later the virus has spread to over 100 countries. Europe and the USA remain the worst affected, with deaths in the UK now having overtaken both Italy and Spain, recording 35,341 deaths by Tuesday 19 May 2020. Life has come to a virtual standstill, with medical services stretched to the limits of normal capacity. It is therefore vital that the medical resources in hospitals are being directed towards caring for the acutely ill with COVID19 , with all medical specialties playing a vital role. While colleagues in Diabetes and Endocrinology (D\&E) are working tirelessly to support frontline services, we still have a duty of care towards those who routinely depend upon D\&E teams to support self-management of their condition. The NHS has issued guidance on how to adapt diabetes services during this crisis. ${ }^{1}$

Clinicians throughout the country have reported that specialist clinical services have been hard hit by COVID which, combined with

Consultant in Diabetes and Endocrinology, Pinderfields Hospital, Wakefield, UK; Chairman ABCD

2 Consultant in Diabetes at King's College Hospital, London, UK; Chair DTN-UK

3 Consultant Diabetologist, University Hospitals of Derby and Burton, Derby UK; Honorary (consultant) Assistant Professor, University of Nottingham, Nottingham, UK

${ }^{4}$ Consultant in Diabetes, Chairman Joint Specialist Committee at RCP, London, UK

Address for correspondence: Dr Dinesh Nagi Diabetes and Endocrine Unit, Pinderfields Hospital, Mid Yorkshire NHS Trust, Wakefield, WF1 4DG, UK

Telephone: 01924213594 E-mail: D.nagi@nhs.net

Br J Diabetes 2020;20:1-4

https://doi.org/10.15277/bjd.2020.246 a worrying reduction in acute admissions, raises concern that those most in need have not sought medical attention. Redeployment of specialist staff to frontline activity has led to many outpatient appointments being cancelled, reducing the support available for people living with diabetes to a bare minimum.

\section{Impact of COVID-19 pandemic on clinical services for Diabetes and Endocrinology (D\&E)}

- To deal with this pandemic, a significant number of specialist medical and nursing staff have been seconded to acute general medical duties and in support of COVID wards. This has created a shortfall of workforce to deal with essential services, with the impact on specialist care disproportionately in smaller units.

- The maintenance of the safe ongoing care of people with diabetes and endocrine disease has undoubtedly been seriously compromised with fewer acute presentations and less access to investigations (including initial face-to-face consultations).

- The loss of standard outpatient clinics and regular day case endocrine investigations.

- There has been a clear reduction in referrals to D\&E departments, raising concerns of important unmet clinical need.

- There are anecdotal reports of late presentations of people with newly diagnosed type 1 diabetes presenting with ketoacidosis and associated harm.

- ST3+ and CMT trainees in D\&E have been seconded solely to acute medical on-call duties and have effectively lost access to specialist training given their current work plans. Their contribution and adaptation to service, however, is worthy of recognition by the NHS.

- In addition to the above, healthcare professionals (HCPs) who have had to work differently, despite their own medical conditions, and the retired HCPs who have volunteered to return and may yet be needed to help the service recovery, are worthy of recognition.

- The current situation is thus forcing delivery of sub-standard care. Although in many cases diabetes MDT foot clinics and antenatal clinics have retained face to face consultations, services have had to adapt to minimise face to face contact. Thus guidelines for the screening of gestational diabetes have abandoned the OGTT in an attempt to minimise risk. 
- People with diabetic foot disease are a high-risk cohort, yet referrals to foot MDT clinics have reached a nadir, perhaps reflecting, in part, a fear from those living with diabetes and, in part, a reluctance for primary care teams to refer to hospital.

- A limited number of services have had access to technology and patient information to deliver these activities from home settings.

The NHS has already started planning the 'rebooting' and recovery of outpatient services, and several specialties including $A B C D$ and the Society for Endocrinology (SfE) have contributed to this work through the Royal College of Physicians (RCP London).

Delivering a 'normal' service going forward will represent a major challenge, where inevitably there will be a significant backlog to deal with. It is likely that several changes to conventional working will be needed to deliver services in the wake of the COVID pandemic. A revolution in diabetes services is called upon to ensure timely responsive care which can address the needs of the individual for the future.

We have the opportunity to redesign models of care to best support people with diabetes whilst undertaking extreme social distancing measures as recommended by the government, not only to contain the spread of COVID-19 but also to protect those at high risk of adverse outcomes. ${ }^{2}$ This is absolutely necessary, and "necessity is the mother of invention".

Over the last few weeks, social media interaction among specialists with diabetes have helped and inspired us to collate alternative effective models of consultations in D\&E, striving to meet the needs of those with diabetes whilst simultaneously deploying limited resources wisely.

The priority during the COVID outbreak has been managing hospital emergencies, supporting the needs of those who are in hospital with diabetes, and facilitating early discharge. There has also been a critical time-dependent need to support pregnant women with diabetes, those with acute foot problems and those with diabetic kidney disease - both renal transplant patients and those undergoing kidney dialysis.

\section{How to move forward beyond the current crisis}

Strategies and innovative ways are needed to support and deliver good quality care to those who are unable to come to routine faceto-face appointments. This requires alternative remote consultations which will require careful organisation, planning and IT support to be able to facilitate the transformation from face-toface to virtual consultations.

$A B C D$ and SfE have produced a report with recommendations to inform the NHS planning, in collaboration with RCP London. This focused on:

(1) Maintaining vital elective care

(2) Beginning to plan the launch of services following disruption

(3) Looking to innovation to change post COVID services

There was a unanimous response from $A B C D$ and SfE membership that, during the recovery phase, current service models for both endocrinology and diabetes require radical change including more use of tele- and video-consultations, new service models, streamlined referral and better integration with primary care.
All current major sub-specialist services for diabetes (young adults, type 1 services, antenatal, feet, complication clinics) will need capacity planned where services were curtailed. Similarly, for general endocrine clinics, additional services may be required with again an expectation that virtual consultations will be required for most reviews.

All sub-specialist services in D\&E will need to relaunch with effective pre-clinic blood tests ( $\mathrm{HbA}_{1 \mathrm{c}}$, renal function, lipids), wider access to and use of specialist nurses, smarter MDT input using video-conferencing and other means of contact.

The approach to tariffs changed in 2020-21, but remains too rigid for the purposes of complex long-term care of diabetes and endocrine disease. Year of care tariffs and new service models that enable quicker and better integrated care between primary and specialist care and additionally between specialist departments for people with complex multi-morbidity are best developed nationally through discussions with the specialist societies.

We need to get our terminology right as well, as this impacts expectation of the consultation, as well as the tariff it attracts. A virtual consultation which replaces a previous face-to-face appointment should attract the same tariff. However, in various settings, 'virtual' clinics can mean either a telephone or email contact, which may be 'ad hoc' in response to a query or planned, or case discussion between HCPs without the patient being present. Ensuring tariffs are optimally structured to support the post-COVID era will be key to ensuring the success of evolving services.

Novel glucose monitoring technology, with cloud-based access to glucose and insulin data, supports virtual consultations. It also allow services to identify those at highest risk and provide targeted care, as well as enhancing the quality of interaction.

Commissioned models should facilitate effective collaborative care between primary and specialist teams without the need for the current time-consuming and unnecessary pattern of referral, triage and standard outpatient consultation for ongoing care. Doing this will require effective IT solutions which enable full access to real-time patient records so that patient information can be accessed and shared seamlessly between primary and specialist teams (such as SystmOne).

It is vital that we ensure that service users are actively involved in any proposed new models of diabetes care, remembering that services should be redesigned 'with service users' rather than 'for them'.

\section{The post COVID-19 revolution in diabetes care}

As we emerge from the COVID-19 pandemic, it is critical that we are in a position to continue to provide specialist diabetes support to those most in need - all people with type 1 diabetes and those with type 2 diabetes with high $\mathrm{HbA}_{1 \mathrm{c}}$ and/or complex co-morbidities.

\section{Face-to-face versus virtual triaging}

There are some consultations that will require face-to-face support; for example, foot examinations, antenatal visits for an ultrasound scan, examination of injection sites and blood tests all require a physical presence. Prescribing or changing medications has typically 
been done in the clinic environment. However, with access to SystmOne, the repeat prescription template can be easily viewed and any changes required can be organised by simply sending a task through to the GP practice.

Virtual clinics are suited to consultations, but there are concerns when it comes to more practical tasks. While some tech-savvy patients (irrespective of age) may be able to perform insulin initiation or start new technology such as an insulin pump or continuous glucose monitoring with virtual support, there will still be a need for some patients to do this face-to-face. Similarly, although traditionally diabetes consultations have included physical measurements of blood pressure or weight, there is an opportunity for the person with diabetes to be supported to undertake these measurements at home where required. Similarly, although we have typically examined injection sites, these can potentially be viewed virtually where a face-to-face review is not possible.

We are aware that, early on in the pandemic, people with diabetes were on a high-risk list, but further risk stratification of the diabetic population has been slow to appear. We recognise that there is a paucity of data nationally to support this and a pragmatic risk stratification will have to be considered, work which is being undertaken by $A B C D$ working closely with the Primary Care Diabetes Society.

As we enter the recovery phase it will become important to triage patients from clinic lists using a traffic light system:

Red: Urgent. An example may be a new diagnosis of type 1 diabetes or insulin initiation in someone with type 2 diabetes and those with symptomatic hyperglycaemia.

Amber: For instance, those with raised $\mathrm{HbA}_{1 c}$, at-risk co-morbidities such as chronic kidney disease, unstable retinopathy, problematic hypoglycaemia or recurrent hospital admissions.

Green: Those with 'stable' diabetes and no acute complications, with access to online support and resources and the ability to contact the team if there is a change in circumstances.

We would like to emphasise that any face-to-face consultations during this ongoing crisis will require appropriate use of personal protective equipment (PPE) by the staff running these clinics, and a system needs to be in place to ensure this while maintaining social distancing measures.

\section{Choosing the right modality for interaction with people with diabetes}

(1) Telephone consultation: This allows useful interaction, is simple, straightforward and supportive for patients. Make sure you provide a time 'window' for the appointment and you have the right number. Often hospital calls come through without caller ID, and so are blocked or the person may not pick up if they are not expecting the call. Ensure the identity of the person you are speaking to and confirm they are in a quiet place where they can speak safely before starting the consultation. Needless to say, there needs to be a record of the clinical interaction.

(2) Video consultation: There are a number of platforms currently available; for example, the 'Attend Anywhere' system is being used by a number of UK trusts. This provides a video consultation with a 'virtual waiting room' for patients to enter before being called into the consultation. This avoids the problem with systems such as Skype of having to generate multiple separate meeting IDs for different patients. These systems enable video calling and allow you to share your screen, which is valuable when discussing results or glucose readings from software such as Diasend or Libreview.

(3) Email: Having a team email that patients are aware of, that is managed and dealt with by a number of members of the team is a valuable way for patients to have a safety net and connect with the team when they need advice. Email is a great way for rapid query resolution or a quick check during dose titrations or adjustments. Most of our specialist nurse colleagues spend a large proportion of time doing emails, and it is important to have a system for documenting and saving that correspondence into electronic patient records for clinical governance as well as for payment for activity.

In addition to a plethora of guidance and advice about remote consultations, the General Medical Council has published guidance for remote consultations and prescribing that should be taken into account. $^{3}$

\section{Choosing the right time for interaction with people with diabetes}

The move to virtual consultations presents an opportunity. While clinics have typically run between 9am and $5 \mathrm{pm}$ to fit with the working hours of the NHS, virtual clinics do not have the same boundaries. There is no need for reception staff to be present; no need for notes to be collected if there is access to electronic records; no need to dictate if there is access to SystmOne to allow the entry to be made directly onto the GP system; and no need for prescriptions if a task can be sent to the GP practice to request a change in medication. Therefore, if the clinical staff are available and willing, then virtual evening clinics could be offered, removing the need for people to take time off work for their diabetes appointments.

\section{Interaction with HCPs}

(1) E-consultations for GP practice support: This is well established as a tool to support GP practices and works exceptionally well given the right systems (eg, SystmOne). Many units have had experience of using SystmOne for some years. The created tasks are dealt with within 48 hours of receipt and specialist advice is recorded in the patient's clinical notes, with a message back to the GP surgery that the query has been dealt with A national tariff is available for this service. It is extremely popular with GPs and can reduce the need for a face-to-face specialist consultation in a significant proportion of cases. We would like to propose that this service needs to be up-scaled for our specialty nationally post-COVID. The time spent by clin- 
icians needs to be job planned. A regular audit of this component of the service is essential to ensure you are keeping up to the agreed standards.

(2) E-consultation for patients: Consultant Connect is a system by which GPs can pose questions to a group of consultants, who can answer the question in real time, often negating the need for a specialist referral and providing a speedy specialist response in real time with the patient often sitting in the primary care physician's office at the time of the call. The only potential disadvantage is that the clinicians answering the call may not be local and may have limited understanding of the local services available to provide ongoing support for the individual if needed. E-mail communication with patients is likely to be across potentially non-secure networks, and patients will need to understand this and give their consent to their HCP using this modality. We hope that this can be taken as implied consent and guidelines need to be adhered to that no sensitive information should be shared in this way.

\section{Data management platforms to support virtual consultations}

(1) Online resources for education and support

As we become more reliant on technology for interaction, we may be able to make more of the online educational and support materials available. The Diabetes Technology Network (DTN) has created a range of educational video content to support both HCPs and patients using continuous glucose monitoring or flash glucose monitoring (https://abcd.care/dtn/education) and are currently working with Glooko and Digibete to create an online platform to help certify HCPs in the use of diabetes technology. The award-winning T1 Resources website (https://www.t1 resources.uk) curates high quality material from the internet and reviews it, providing a safe place for patients to access information. There is a wealth of information on websites such as Bertie-online (https://www.bertieonline.org.uk), TREND (https://trend-uk.org) and, of course, the ABCD website, as well as product-specific information from the websites of diabetes technology companies.

\section{What are the challenges among all this?}

Perhaps the biggest challenge is to enable acute trusts, CCGs and PCNs to change and adapt new models of care, tariffs and ensure full dedicated IT support, with consensus from primary care.

Extra administrative support is needed to support the planning and implementation of the recovery phase with or without using the new ways of consultation.

It is anticipated that new patient referrals in D\&E will need additional clinics to be established, given the current low referral rates. The major backlog of case management will require substantial additional specialist medical and nursing time devoted to catchup care, and we may be counting the human cost of the COVID-19 pandemic for years to come.

We need to be aware of the likely impact of these changes on

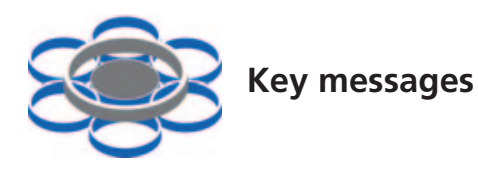

- Outpatient Diabetes services have been severely disrupted during the COVID-19 pandemic and the impact on smaller diabetes units may be significant

- The recovery of lost activity will be challenging, but needs to start immediately, with risk stratification, as to which patients with diabetes should be seen first and requiring urgent support both in primary care and under specialist teams

- We suggest, that Diabetes teams learn from the innovation and new technologies we have used during this crisis, to build better models of care, which must involve carers

trainees in the specialty, and this needs discussing and adapting to ensure that specialist training is not compromised but enhanced. We must not forget the physical and emotional impact of frontline management of COVID-19 on our medical and nursing teams, and this will need to be taken into account with any rescheduled activity.

Furthermore, the emotional needs of the people we see in clinic need to be considered. We typically rely on non-verbal cues to guide our communication style and content in face-to-face consultations. This is almost certainly more challenging during virtual consultations, where direct eye contact may not be possible and subtle non-verbal cues might be missed. Consideration of how we can best meet the psychosocial needs of people with diabetes in this new virtual world will require some thought.

An additional challenge is how to deal with the language barrier to provide virtual consultations to those whose first language is not English.

\section{Conclusions}

We believe that during the COVID-19 crisis the NHS has been responsive, and has shown a willingness to modernise more in the past five weeks than it has in the past five years and, in many instances, better than before. However, lots more still needs to be done. Clinicians and patients are supportive of the changes. There is no going back. This is likely to be the new normal.

\section{Conflict of interest None Funding None}

\section{References}

1. NHS. Diabetes COVID-19 key information. https://www.england.nhs.uk/ london/london-clinical-networks/our-networks/diabetes/diabetes-covid19-key-information/

2. GOV.UK. Staying alert and safe (social distancing). https://www.gov.uk/ government/publications/staying-alert-and-safe-social-distancing

3. General Medical Council. Remote prescribing high level principles. https://www.gmc-uk.org/ethical-guidance/learning-materials/remoteprescribing-high-level-principles 